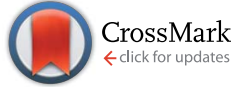

Cite this: Soft Matter, 2014, 10, 6546

Received 15th March 2014

Accepted 17th June 2014

DOI: $10.1039 / \mathrm{c} 4$ sm00577e

www.rsc.org/softmatter

\title{
Transient dynamics during stress overshoots in binary colloidal glasses
}

\author{
T. Sentjabrskaja, ${ }^{\text {a }}$ M. Hermes, ${ }^{\text {b }}$ W. C. K. Poon, ${ }^{\text {b }}$ C. D. Estrada, ${ }^{c}$ R. Castañeda-Priego, ${ }^{c}$ \\ S. U. Egelhaaf ${ }^{a}$ and M. Laurati ${ }^{\star a}$
}

We investigate, using simultaneous rheology and confocal microscopy, the time-dependent stress response and transient single-particle dynamics following a step change in shear rate in binary colloidal glasses with large dynamical asymmetry and different mixing ratios. The transition from solid-like response to flow is characterised by a stress overshoot, whose magnitude is linked to transient superdiffusive dynamics as well as cage compression effects. These and the yield strain at which the overshoot occurs vary with the mixing ratio, and hence the prevailing caging mechanism. The yielding and stress storage are dominated by dynamics on different time and length scales, the short-time incage dynamics and the long-time structural relaxation respectively. These time scales and their relation to the characteristic time associated with the applied shear, namely the inverse shear rate, result in two different and distinct regimes of the shear rate dependencies of the yield strain and the magnitude of the stress overshoot.

\section{Introduction}

A wide range of technical applications is based on glassy materials, including polymeric, ${ }^{1}$ metallic $^{2}$ and colloidal systems. $^{3}$ One-component dispersions of hard-sphere like colloids have been intensively used as model systems to study the glass transition. ${ }^{3}$ In this system, the volume fraction $\phi$ is the only control parameter. The glass state is driven by crowding: for $\phi>0.58$ particles are permanently localised in cages formed by their neighbours, which they can only escape through activated processes. ${ }^{4}$ Colloidal glasses melt and flow under application of shear. ${ }^{5-13}$ Shear-induced melting is associated with an irreversible deformation of the cage $e^{9,13}$ and the onset of diffusive dynamics. ${ }^{8}$ It occurs via a transient regime in which the system yields. At yielding a stress overshoot is observed in the rheological response and reflects maximal cage distortion in the structure and a transient super-diffusive regime in the dynamics. ${ }^{9,13-15}$

Many glassy materials used in applications are not onecomponent systems, but composed of particles with different sizes. This raises the question whether, and if so how, the shearinduced melting process, in particular the transient macroscopic rheology and the microscopic structure and dynamics, is affected by the presence of multiple components. The simplest

${ }^{a}$ Condensed Matter Physics Laboratory, Heinrich Heine University, Universitätsstraße 1, 40225 Düsseldorf, Germany. E-mail: marco.laurati@uni-duesseldorf.de

${ }^{b}$ SUPA, School of Physics \& Astronomy, The University of Edinburgh, Mayfield Road, Edinburgh EH9 3JZ, UK

${ }^{c}$ Division of Sciences and Enginering, University of Guanajuato, Loma del Bosque 103, 37150 León, Mexico multi-component model system is a binary mixture of colloidal hard spheres. The phase behavior of binary colloidal hard spheres has been studied in experiments, ${ }^{16-20}$ simulations $^{21-23}$ and theory. ${ }^{23-29}$ It depends on several parameters, namely the total volume fraction, the size ratio and the mixing ratio of the two components. Theory predicts that at small to moderate size disparities the glass transition shifts to larger total volume fractions, similar to the effect of polydispersity. ${ }^{24,30-32}$ This implies that for constant total volume fraction, glass melting can be induced by mixing. This is reflected in the acceleration of the dynamics measured by light scattering ${ }^{16}$ as well as the strong reduction of the viscosity observed by rheology. ${ }^{33}$ At large enough size disparities multiple glass states are expected..$^{30}$ They differ by the mechanism driving the arrest of the large spheres, either caging or depletion-induced bonding, and the dynamics of the small spheres, either dynamical arrest or mobility. ${ }^{25,30}$ Some of these states have been observed experimentally ${ }^{17-19}$ and in molecular dynamics simulations. ${ }^{21}$

The yielding behaviour of binary glasses under oscillatory shear was recently studied for size ratios $\delta=R_{\mathrm{S}} / R_{\mathrm{L}}=0.38$ and $0.2,{ }^{20}$ with $R_{\mathrm{S}}$ and $R_{\mathrm{L}}$ the radii of the small and large spheres respectively. At constant total volume fraction $\phi$, a decrease of the yield strain and stress is observed at intermediate mixing ratios, and is particularly pronounced for the larger size disparity. This effect has been associated with the variation in the free volume due to changes in the volume fraction of random close packing, which also becomes more pronounced at larger size disparities.

Here, we extend this study to explore the response after switch-on of a constant shear rate. In particular the link 
between the macroscopic non-linear rheology and the transient single-particle dynamics is investigated using confocal microscopy. A stress overshoot and super-diffusive transient dynamics is found to characterise yielding, similar to the behaviour of one-component systems.,.$^{\mathbf{9} 13-15}$ However, in binary mixtures the yield strain and magnitude of the overshoot depend in a complex and different way on the shear rate and show a dependence on the composition of the mixture. The composition determines the caging mechanism, localization length as well as the short and long-time dynamics, including the degree of super-diffusion.

The manuscript is structured as follows. Section 2 describes the experimental systems and methods, namely simultaneous rheology and confocal microscopy, as well as the simulations. In Section 3 we first present the equilibrium structure and dynamics of the large particles in the mixtures and a resume of the linear viscoelastic properties of the binary mixtures. Then we discuss the results of the non-linear rheology and the dynamics under shear before offering some conclusions in Section 4 .

\section{Methods}

\subsection{Rheology}

Rheological measurements are performed using an ARES G2 strain controlled rheometer (TA instruments) with a cone-plate geometry (diameter $20 \mathrm{~mm}$, cone angle $2^{\circ}$, truncation gap 0.054 $\mathrm{mm}$ ). A solvent trap minimizes solvent evaporation. Rheological measurements on colloidal glasses can be affected by loading effects, shear history and aging. Therefore, before each test a renjuvenation procedure is performed in order to obtain a reproducible initial state. First, after loading we perform a dynamic strain sweep to estimate the yield strain $\gamma_{\text {yield }}$ of the system. Oscillatory shear at strain amplitude $\gamma=300 \% \gg \gamma_{\text {yield }}$ is applied to induce flow and maintained until the viscoelastic storage, $G^{\prime}$, and loss, $G^{\prime \prime}$, moduli reach a stationary state, typically after $200 \mathrm{~s}$. Afterwards, oscillatory shear in the linear viscoelastic regime $(0.05 \%<\gamma<0.1 \%$, depending on sample) is applied until $G^{\prime}$ and $G^{\prime \prime}$ become stationary, typically for times $200 \mathrm{~s}<t<700 \mathrm{~s}$, depending on the sample. The state characterised by the stationary values of $G^{\prime}$ and $G^{\prime \prime}$ thus represents the initial reproducible state. The absence of wall slip is verified by comparison with measurements obtained with roughened geometries (data not shown).

\subsection{Confocal microscopy under shear}

Confocal microscopy measurements under shear are performed with a confocal rheoscope, which is a combination of an MCR301 WSP rheometer (Anton Paar) and a fast-scanning VT-Eye confocal scanner (Visitech), mounted on a Nikon Ti-U inverted microscope with a Nikon Plan Apo $60 \times$ objective (NA $=1.40$ ). Details of the setup can be found in previous work. ${ }^{34}$ We use a cone-plate geometry with diameter $50 \mathrm{~mm}$, cone angle $1^{\circ}$ and truncation gap $0.10 \mathrm{~mm}$. To minimise wall-slip the cone is sandblasted, while the bottom plate, consisting of a thin glass plate, is coated with PMMA particles of size
$0.885 \mu \mathrm{m}$ and $0.174 \mu \mathrm{m} .{ }^{35}$ A solvent trap is used to reduce solvent evaporation. Images of the samples $(512 \times 512$ pixels, corresponding to about $48 \mu \mathrm{m} \times 48 \mu \mathrm{m}$ for samples with $0.3<$ $x_{\mathrm{s}}<0.9,51 \mu \mathrm{m} \times 51 \mu \mathrm{m}$ for $x_{\mathrm{s}}=0.0$, and $53 \mu \mathrm{m} \times 53 \mu \mathrm{m}$ for $x_{\mathrm{s}}=0.1$ ) are acquired at a depth of $30 \mu \mathrm{m}$ from the bottom plate and at a distance of about $6 \mathrm{~mm}$ from the center. Time series of $2 \mathrm{D}$ images are taken at a rate of 31 or 67 frames per second, depending on the sample. Particle coordinates and trajectories are extracted from the pictures using previouslyexplained routines. ${ }^{36}$

\subsection{Samples}

We use suspensions of polymethylmethacrylate (PMMA) colloids, sterically stabilized with polyhydroxystearic acid (PHS) and dispersed in a solvent mixture of cis-decalin and cycloheptyl bromide (CHB). The solvent mixture matches the density and almost the refractive index of the particles. The charge that the particles acquire in the $\mathrm{CHB} /$ decalin solvent is screened by adding $4 \mathrm{mM}$ tetrabutylammoniumchloride (TBAC). ${ }^{37}$ Under these conditions the interactions in the system are hard-spherelike. ${ }^{38}$ For the most sensitive rheological measurements we use particles with radii $R_{\mathrm{L}}^{\text {rheo }}=0.304 \mu \mathrm{m}$ and $R_{\mathrm{s}}^{\text {rheo }}=0.063 \mu \mathrm{m}$, and polydispersities of approximately $10 \%$ and $15 \%$, respectively. The size ratio of the mixture is $\delta^{\text {rheo }}=0.207$. The high energy density of these small particles leads to a strong rheological signal. The sample set corresponding to these particles is referred to as $\mathrm{RH}$ in the following. For measurements on the confocal rheoscope, a mixture of PMMA particles with radii $R_{\mathrm{L}}^{\mathrm{mic}}=0.885 \mu \mathrm{m}(6 \%$ polydispersity $)$ and $R_{\mathrm{S}}^{\mathrm{mic}}=0.174 \mu \mathrm{m}(15 \%$ polydispersity) is prepared resulting in $\delta^{\text {mic }}=0.197$. The large spheres with radius $R_{\mathrm{L}}^{\text {mic }}$ are fluorescently labelled with nitrobenzoxadiazole (NBD) and can be observed with the confocal microscope using a solid state laser with wavelength $\lambda=488$ $\mathrm{nm}$. This sample set is referred to as CO in the following. The particle radii and polydispersities are determined by static and dynamic light scattering with an uncertainty in the radius of about $2 \%$.

The volume fraction of the sediment of the large spheres is determined by imaging the sample by confocal microscopy and using the Voronoi construction to estimate the mean Voronoi volume per particle. The procedure of determining the volume fraction is described in detail $\mathrm{in}^{20}$ and leads to the estimate $\phi_{\mathrm{L}}^{\mathrm{RCP}} \approx 0.68$. A one-component sample with $\phi=0.61$ is prepared by diluting the sediment. This sample is used as a reference. The volume fractions of the samples containing the small particles are adjusted in order to obtain comparable linear viscoelastic moduli in units of the energy density $3 k_{\mathrm{B}} T /$ $4 \pi R^{3}$, where $k_{\mathrm{B}}$ is the Boltzmann constant, $T$ the temperature and $R$ the particles' radius, while multiplying the frequency by the free-diffusion Brownian time $\tau_{0}=6 \pi \eta R^{3} / k_{\mathrm{B}} T$, where $\eta=$ $2.2 \mathrm{mPa} \mathrm{s}$ is the solvent viscosity. In this way we obtain samples with comparable dynamics, according to the generalised Stokes-Einstein relation. ${ }^{39}$ Samples with constant total volume fraction $\phi=0.61$ and different compositions, namely fractions of small particles $x_{\mathrm{s}}=\phi_{\mathrm{s}} / \phi$, where $\phi_{\mathrm{s}}$ is the volume fraction of small particles, are prepared by mixing the stock solutions. 


\subsection{Simulations}

Event-driven molecular dynamics simulations are performed to investigate the dynamics of binary hard spheres with the same size ratio $\delta=0.2$ as in the experiments. To render simulations with this size disparity feasible, we applied the double-cell scheme, ${ }^{23}$ which uses a combination of large cells with a size just above $2 R_{\mathrm{L}}$ and small cells with a size just above $2 R_{\mathrm{S}}$. This allows us to compute long enough sequences of particle configurations. Due to the nature of the hard-sphere potential, the system is conservative and additionally the temperature is constant. Thus, its evolution can be determined by calculating a sequence of elastic collisions; the particles move in a straight line before any collision. Given the positions, $\vec{r}_{i}$, and velocities, $\vec{v}_{i}$, of each pair $(i, j)$ of particles at time $t$, the collision time $\Delta t$ is determined by the physical solution (real and positive) of the quadratic equation $\vec{r}_{i j}^{2}(t+\Delta t)=\left[\vec{r}_{i j}(t)+\vec{v}_{i j}(t) \Delta t\right]^{2}=\left[\left(2 R_{i}+2 R_{j}\right) / 2\right]^{2}$. The set of collision times of each particle is stored in an ordered list to monitor its trajectory with a nonuniform time step sequence. In each collision, the change in the velocities of the colliding particles is obtained by the energy and momentum conservation laws as $\Delta \vec{v}_{i}=-2 m_{j}\left(\vec{v}_{i j} \cdot \vec{r}_{i j}\right) \hat{r}_{i j} /\left(m_{i}+m_{j}\right)$. Hence, the next collision can be predicted. Thus, the simulations provide particle trajectories, based on which the mean squared displacement can be determined, as well as, e.g., the mean free path $l_{0}$ and the mean time between collisions, $\mathscr{T}_{\mathrm{s}}^{\text {short }}$. With increasing volume fraction, $\mathscr{T}_{\mathrm{s}}^{\text {short }}$ approaches zero and thus the rate of collisions quickly grows. With our computing resources we can investigate volume fractions $\phi \leq 0.58$, i.e. below the experimental volume fraction. Experiments with $\phi=0.61$ (Fig. 2) and $\phi=0.58$ (ref. 40) indicate that the qualitative variations of the dynamics, quantified by the mean squared displacements, as a function of mixing ratio are comparable for the two volume fractions. We thus compare our experimental findings to simulation results for $\phi=0.58$. The simulations cover $0.1 \leq x_{\mathrm{s}} \leq 0.7$ and the one-component limits $x_{\mathrm{s}}=0.0$ and 1.0. The numbers of large particles are $125\left(x_{\mathrm{s}}=0.7\right), 250\left(x_{\mathrm{s}}=\right.$ 0.5 ), 500 (other $x_{\mathrm{s}}$ ) and according numbers of small particles. The large and small spheres have the same mass density and the two populations are monodisperse. The simulations start with random particle configurations. At least 10 different runs are averaged for each $x_{\mathrm{s}}$ to reduce statistical uncertainties.

\section{Results and discussion}

\subsection{Quiescent structure}

Binary mixtures with a size ratio $\delta=0.2$, a total volume fraction $\phi=0.61$ and different compositions $0 \leq x_{\mathrm{s}} \leq 1$ are investigated. The pair distribution functions $g(r)$ of the large particles in the quiescent state were determined by confocal microscopy (Fig. 1). They indicate an amorphous structure for all $x_{\mathrm{s}}$. Similar data were reported and discussed in detail in ref. 19 . We thus only recall the main findings. The one-component glass of large spheres shows a fluid-like structure typical of a colloidal glass; a main peak corresponding to the first shell of nearest neighbours at distance $r=2 R_{\mathrm{L}}$ (the caging particles) and additional peaks indicating the successive shells of nearest neighbours.

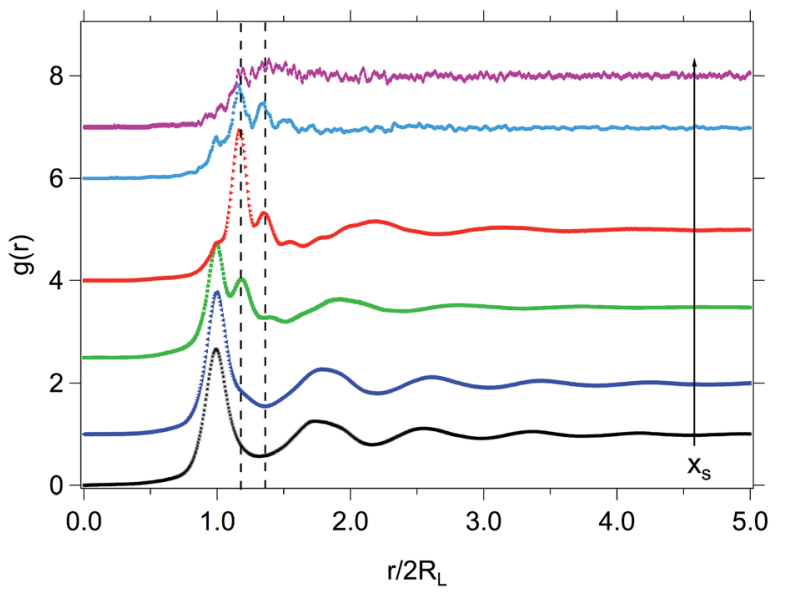

Fig. 1 Pair distribution function $g(r)$ of large particles $R_{\mathrm{L}}^{\text {mic }}$ in mixtures with $\phi=0.61, \delta=0.2$ and different compositions $x_{\mathrm{s}}=0.0(*), 0.1(<)$, $0.3(\square), 0.5(\downarrow), 0.7(\bullet), 0.9(\diamond)$. Data for $x_{\mathrm{s}}>0$ are shifted vertically. Dashed lines indicate particle-particle distances $r=2\left(R_{\mathrm{L}}+R_{\mathrm{s}}\right)$ and $r=$ $2\left(R_{\mathrm{L}}+2 R_{\mathrm{S}}\right)$, corresponding to configurations in which two large particles are separated by one or two small particles, respectively.

Upon addition of small spheres, additional particle configurations appear due to the intercalation of small spheres between large spheres. While a small shoulder at $r=2 R_{\mathrm{L}}+2 R_{\mathrm{S}}$ is already visible for $x_{\mathrm{S}}=0.1$, peaks at this distance and also at $r=2 R_{\mathrm{L}}+$ $4 R_{\mathrm{s}}$ are observed for $x_{\mathrm{s}}=0.3$, which correspond to configurations in which two large particles are separated by one or two small particles, respectively (Fig. 1, dashed lines). This indicates a loosening of the cage of large particles with increasing $x_{\mathrm{s}}$, which leads to a transition in caging at $x_{\mathrm{s}}=0.5$, as indicated by the disappearing first peak at $r=2 R_{\mathrm{L}}$ and the pronounced peak at $r=2 R_{\mathrm{L}}+2 R_{\mathrm{s}}$. Hence, at $x_{\mathrm{s}}=0.5$ the large spheres are prevalently caged by small spheres. Upon further increasing $x_{\mathrm{s}}$ the large particles, still caged by small particles, become increasingly more dilute. Particle configurations in which small particles intercalate between large particles were not observed in mixtures with larger $\delta=0.67,{ }^{41}$ in agreement with geometrical arguments $^{20}$ predicting a limiting value $\delta \leq 0.41$.

\subsection{Quiescent dynamics}

The mean squared displacement (MSD) of the large particles in one direction is:

$$
\delta y^{2}(t)=\left\langle\left(y_{i}\left(t+t_{0}\right)-y_{i}\left(t_{0}\right)\right)^{2}\right\rangle_{i, t_{0}},
$$

where $t$ is the delay time, $t_{0}$ a selected time along the trajectory of particle $i$ and \langle\rangle$_{i, t_{0}}$ indicates the average over all particles $i$ in the field of view and all times $t_{0}$. It is determined from time series of 3D stacks in the quiescent state before applying shear (Fig. 2). For $x_{\mathrm{s}}=0.0$ and 0.1 the MSDs are flat, indicating localisation of particles in cages and absence of long-time diffusion within the measurement window. The localisation length $L=\sqrt{\delta y^{2}\left(t_{1}\right)}$, with $t_{1}$ the shortest delay time measured, corresponds to that expected for a cage of large particles. For $x_{\mathrm{s}}=0.3$ the large-particle dynamics become diffusive at long times. Similarly, for $x_{\mathrm{s}}=0.5$ mobility is observed at long times 


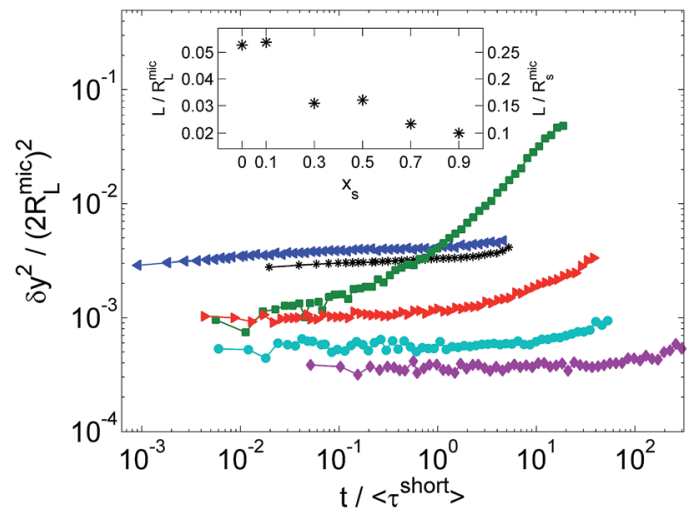

Fig. 2 Quiescent mean squared displacement in one direction $\delta y^{2}$ of large particles $R_{\mathrm{L}}^{\mathrm{mic}}$ in mixtures with $\phi=0.61, \delta=0.2$ and different compositions $x_{\mathrm{s}}=0.0(*), 0.1(\Delta), 0.3(\square), 0.5(\downarrow), 0.7(\bullet), 0.9(\diamond)$. The delay time $t$ is normalised by the composition-averaged short-time Brownian time $\left\langle\tau^{\text {short }}\right\rangle$. (Inset) The $x_{s}$-dependence of the localisation length $L=\sqrt{\delta y^{2}\left(t_{1}\right)}$ in units of $R_{\mathrm{L}}^{\text {mic }}$ (left $y$-axis) and $R_{\mathrm{s}}^{\text {mic }}$ (right $y$-axis), where $t_{1}$ is the shortest delay time measured.

even though no diffusive regime is visible within the experimental time window. In addition, the localisation length $L$ is reduced, indicating the presence of small particles around the large particles, hindering their motions. This is consistent with the pair distribution function of the large particles (Fig. 1), which shows an increasingly more pronounced shoulder at a distance corresponding to the sum of a large and small particle. ${ }^{19,20}$ For larger fractions of small particles, $x_{\mathrm{s}}>0.5$, the long-time dynamics again slow down and particles continue to become increasingly localised in the cage of small particles. This transition in caging and the faster dynamics at intermediate compositions have been observed previously for the same $\delta .{ }^{19}$ However, the acceleration of the dynamics in the present mixtures is much more pronounced than at larger $\delta .^{16,20,31,32,42,43}$ This could result from the melting of the cage of large spheres, which accompanies the glass-glass transition observed at $x_{\mathrm{s}}=$ 0.5 in our system. This appears to affect the particle dynamics more than the smaller cage polydispersity in mixtures of particles with more comparable sizes. Furthermore, the dependence of the MSD on $x_{\mathrm{s}}$ can be related to the available free volume in the mixtures, which can be estimated on the basis of the $x_{\mathrm{s}}$ dependence of the volume fraction of random close packing, $\phi_{\mathrm{RCP}}{ }^{19,20}$

The intrinsic time scales of the samples can be obtained from the corresponding short- and long-time diffusion coefficients. The short-time Brownian time of the small particles, $\tau_{\mathrm{s}}^{\text {short }}=R_{\mathrm{s}}{ }^{2} / D_{\mathrm{s}}^{\text {short }}$ with the short-time diffusion coefficient $D_{\mathrm{s}}^{\text {short }}=f D_{0, \mathrm{~s}}$. It is related to the free (dilute) diffusion coefficient $D_{0, \mathrm{~s}}=k_{\mathrm{B}} T / 6 \pi \eta R_{\mathrm{s}}$ by the $\phi$-dependent factor $f$. In a one-component system, $f$ can be estimated by extrapolating the data in Fig. 8 of ref. 44 to $\phi=0.61$, yielding $f \approx 1 / 32$. Similarly, the short-time Brownian time of the large particles, $\tau_{\mathrm{L}}^{\text {short }}=\tau_{\mathrm{s}}^{\text {short }} / \delta^{3}$, can be determined. For binary mixtures, the compositionaveraged short-time Brownian time in the dilute limit is $\left\langle\tau_{0}^{\text {short }}\right\rangle=6 \pi \eta\left\langle R^{3}\right\rangle / k_{\mathrm{B}} T$ and at a volume fraction $\phi$ we obtain $\left\langle\tau^{\text {short }}\right\rangle=\left\langle\tau_{0}^{\text {short }}\right\rangle / f$, where $\left\langle R^{3}\right\rangle=R_{\mathrm{L}}{ }^{3} /\left[1-x_{\mathrm{s}}\left(1-1 / \delta^{3}\right)\right]$ is the number-averaged cube of the radius.
We studied the long-time dynamics using event-driven molecular dynamics simulations of binary mixtures of hardspheres $^{23}$ with the same size ratio $\delta=0.2$, but a reduced total volume fraction $\phi=0.58$ to keep the simulation times reasonable (Section 2.4). Although the simulations do not consider a solvent and thus do not include Brownian motion at short times, an effective short-time diffusion coefficient $D_{\mathrm{s}}^{0}$ can be determined; $D_{\mathrm{s}}^{0}=l_{0}^{2} / \mathscr{T}_{\mathrm{s}}^{\text {short }}$ with the mean free path $l_{0}$ and mean free time $\mathscr{T}_{\mathrm{s}}^{\text {short }}{ }^{45}$ With this rescaling the ratio $D_{\mathrm{s}}^{*}$ is equivalent to that obtained in a system with Brownian dynamics; $D_{\mathrm{s}}^{*}=$ $D_{\mathrm{s}}^{\text {long }} / D_{\mathrm{s}}^{\text {short }}$, with $D_{\mathrm{s}}^{\text {short }}$ the short-time Brownian diffusion coefficient. ${ }^{45}$ The same equivalence applies to the ratio of the long time relaxation time $\mathscr{T}_{\mathrm{s}}^{\text {long }}$ and the mean free time $\mathscr{T}_{\mathrm{s}}^{\text {short }}$. Then $D_{\mathrm{s}}^{*}$ for the small (and, similarly, the large) spheres can be extracted from the MSDs rescaled by $l_{0}^{2}$ with times rescaled by $\mathscr{T}_{\mathrm{s}}^{\text {short }}$. To simplify the comparison with experiments, in what follows we will indicate the ratio $\mathscr{T}_{\mathrm{s}}^{\text {long }} / \mathscr{T}_{\mathrm{s}}^{\text {short }}$ using the equivalent ratio of the Brownian relaxation times $\tau_{\mathrm{s}}^{\text {long }} / \tau_{\mathrm{s}}^{\text {short }}$. From $D_{\mathrm{s}}^{*}$, the normalised long-time structural relaxation time of the small spheres, $\tau_{\mathrm{s}}^{\text {long }} / \tau_{\mathrm{s}}^{\text {short }}=1 / D_{\mathrm{s}}^{*}$, and, similarly, of the large spheres, $\tau_{\mathrm{L}}^{\text {long }} / \tau_{\mathrm{s}}^{\text {short }}=1 /\left(\delta^{3} D_{\mathrm{L}}^{*}\right)$, can be calculated (Fig. 3 ).

The structural relaxation time of the small spheres, $\tau_{s}^{\text {long, }}$ monotonously increases with $x_{\mathrm{s}}$ indicating the progressive arrest of the small spheres. However, the structural relaxation time of the large spheres, $\tau_{\mathrm{L}}^{\text {long }}$, exhibits an intermediate minimum $\left(x_{\mathrm{s}}=0.1\right)$ consistent with the melting of the onecomponent glasses as a second species is added. While the addition of small spheres to the glass of large spheres melts the glass, the addition of large spheres not only melts the glass of small spheres, but also induces obstacles. ${ }^{46}$ This leads to the asymmetric dependence of $\tau_{\mathrm{L}}^{\text {long }}$ on $x_{\mathrm{s}}$. We expect the minimum to be more pronounced for the higher $\phi=0.61$ of the experiments, since the large and small spheres are deeper in the glassy state at $x_{\mathrm{s}}<0.3$ and $x_{\mathrm{s}} \geq 0.7$ than at $\phi=0.58$. Previous experimental work on binary mixtures with the same size ratio and comparable $x_{\mathrm{s}}=0.7$ indicates glass states for $\phi>0.57$ and

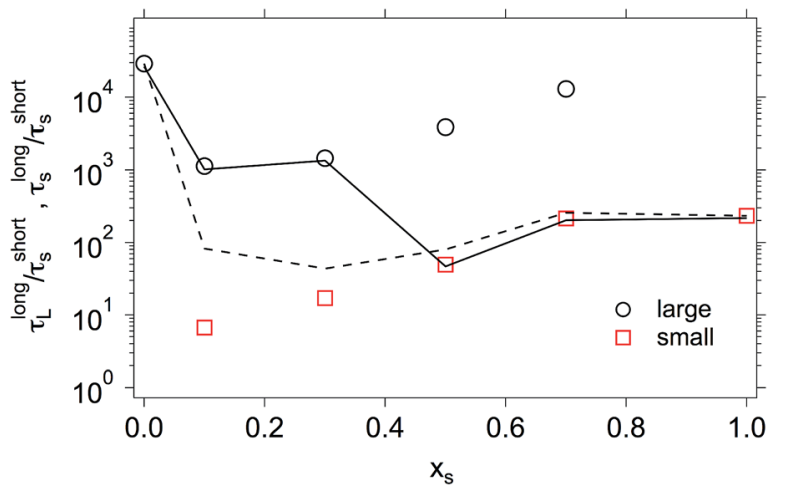

Fig. 3 Long-time structural relaxation times of large, $\tau_{L}^{\text {long }}(0)$, and small, $\tau_{s}^{\text {long }}(\square)$, spheres as a function of composition $x_{s}$, obtained from MD simulations of binary hard sphere mixtures with size ratio $\delta=0.2$ and total volume fraction $\phi=0.58$. The relaxation times are normalised by the mean free time of the small spheres $\tau_{s}^{\text {short }}$. The dashed and solid lines indicate the number-averaged, $\left\langle\tau^{\text {long }}\right\rangle$, and dominant, $\tilde{\tau}^{\text {long, }}$, structural relaxation times, respectively. 
fluid states for $\phi \leq 0.57 .^{20}$ In addition, the number-averaged long-time structural relaxation time at a volume fraction $\phi=$ 0.58 can be calculated according to $\left\langle\tau^{\text {long }}\right\rangle=\left[\left(1-x_{\mathrm{s}}\right) \delta^{3} \tau_{\mathrm{L}}^{\text {long }}+\right.$ $\left.x_{\mathrm{s}} \tau_{\mathrm{s}}^{\text {long }}\right] /\left[\left(1-x_{\mathrm{s}}\right) \delta^{3}+x_{\mathrm{s}}\right]$ (Fig. 3, dashed line). This exhibits a minimum at $x_{\mathrm{s}} \approx 0.3$. The minimum is shifted with respect to the minimum of $\tau_{\mathrm{L}}^{\text {long }}\left(x_{\mathrm{s}} \approx 0.1\right)$ due to the increasing weight of the smaller $\tau_{\mathrm{s}}^{\text {long }}$. As mentioned above, a transition in caging is expected at $x_{\mathrm{s}} \approx 0.5$ with caging by large and small spheres at small and large $x_{\mathrm{s}}$, respectively. ${ }^{20}$ Thus, the systems are expected to be dominated by $\tau_{\mathrm{L}}^{\text {long }}$ and $\tau_{\mathrm{s}}^{\text {long }}$ for $x_{\mathrm{s}} \lesssim 0.5$ and $x_{\mathrm{s}} \gtrsim 0.5$, respectively, which we denote by $\tilde{\tau}_{\mathrm{s}}^{\text {long }}$ (Fig. 3, solid line).

\subsection{Linear viscoelasticity}

The storage modulus, $G^{\prime}$, as a function of composition $x_{\mathrm{s}}$ is extracted from the linear viscoelastic regime of dynamic strain sweeps $\left(0.5 \%<\gamma<1 \%\right.$, depending on sample), Fig. 4 . Values of $G^{\prime}$ are determined for an oscillatory Péclet number $\mathrm{Pe}_{\omega}=1.2$ with $\mathrm{Pe}_{\omega}=\omega\left\langle\tau^{\text {short }}\right\rangle$, where $\omega$ is the oscillation frequency. They are reported in units of the composition-averaged energy density, $k_{\mathrm{B}} T /\left\langle R^{3}\right\rangle$, to remove the trivial dependence on the particle size. The large values of $G^{\prime}$ at $x_{\mathrm{s}}=0.0$ and 1.0 are consistent with their one-component glass states. By adding a second species, $G^{\prime}$ decreases, indicating glass softening with the results for both sample sets, RH (radii $0.304 \mu \mathrm{m}, 0.063 \mu \mathrm{m}$ ) and CO (radii $0.885 \mu \mathrm{m}, 0.174 \mu \mathrm{m}$ ) being comparable. The glass softening is thought to result from the transition in caging and the faster long-time dynamics at intermediate compositions (Fig. 2). ${ }^{19}$ It is particularly pronounced for $0.1 \leq x_{\mathrm{s}} \leq 0.5$, i.e. upon adding small particles to large particles. This reflects the asymmetry observed in the dynamics. The dependence of $G^{\prime}$ on $x_{\mathrm{s}}$ hence appears related to changes in the microscopic dynamics. ${ }^{\mathbf{1 9 , 2 0}}$

\subsection{Non-linear stress response}

In a step rate experiment, a constant shear rate $\dot{\gamma}$ is applied to the initially quiescent sample and the evolution of the stress $\sigma$ as a function of time $t$ or, equivalently, strain $\gamma=\dot{\gamma} t$ is measured. The dependence of the measured stress on strain is presented in Fig. 5 for binary mixtures with size ratio $\delta=0.2$, total volume fraction $\phi=0.61$ and different compositions $x_{\mathrm{s}}$ as

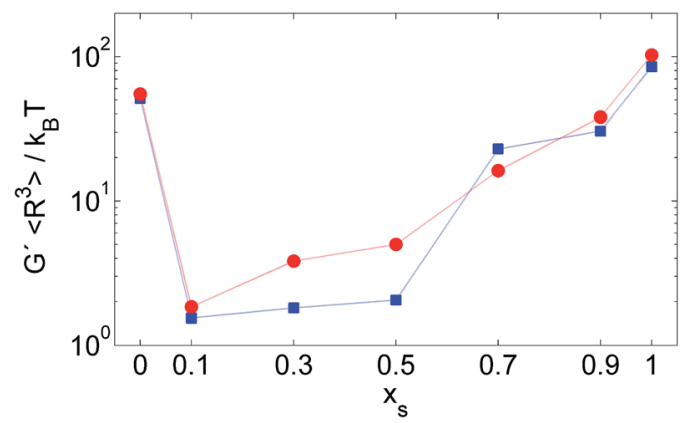

Fig. 4 Storage modulus $G^{\prime} /\left(k_{\mathrm{B}} T /\left\langle R^{3}\right\rangle\right)$ in the linear viscoelastic regime, extracted from dynamic strain sweep measurements at oscillatory Péclet numbers $\mathrm{Pe}_{\omega}=1.2$ for two sample sets with $\phi=0.61, \delta=0.2$ : (•) $\mathrm{CO}$ (larger spheres, also used for microscopy) and $(\square) \mathrm{RH}$ (smaller spheres, only used for rheology).

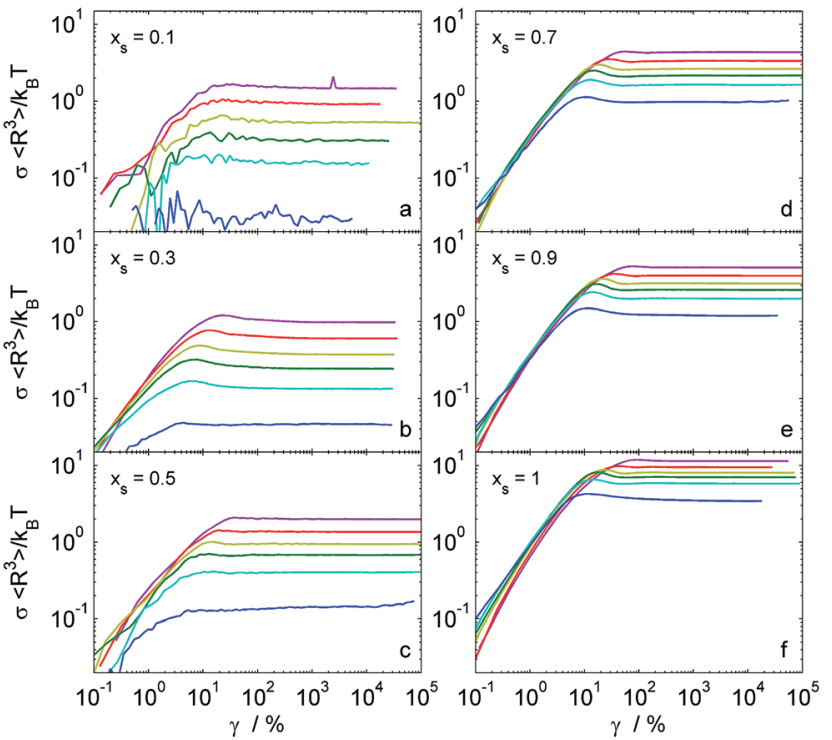

Fig. 5 Stress $\sigma$ scaled by the average energy density $k_{\mathrm{B}} T /\left\langle R^{3}\right\rangle$ vs. strain $\gamma$ measured in step rate experiments for samples with compositions $x_{5}$ (as indicated) and Péclet numbers $\mathrm{Pe}_{\dot{\gamma}}=0.03,0.24,0.64,1.20,2.40$ and 4.70 (bottom to top).

well as different shear rates $\dot{\gamma}$ or Péclet numbers $\mathrm{Pe}_{\dot{\gamma}}=\dot{\gamma}\left\langle\tau^{\text {short }}\right\rangle$. For these values of $\mathrm{Pe}_{\dot{\gamma}}$ and $\phi$, homogeneous flow, i.e. laminar flow in the absence of shear banding, is expected for one component systems. ${ }^{35,47}$ In order to compare different mixing ratios, the stress $\sigma$ is scaled by the composition-averaged energy density. For all $x_{\mathrm{s}}$ and $\mathrm{Pe}_{\dot{\gamma}}$, at small strains $\gamma$ the stress increases almost linearly and reaches a maximum or overshoot, $\sigma_{\text {peak }}$, at a strain $\gamma_{\text {peak }}$. Subsequently the stress decreases to a constant value, $\sigma_{\text {steady }}$, which is the steady state value of the stress when the system flows. The noise in the measurements is seen to decrease with increasing $x_{\mathrm{s}}$ as a result of the increasingly larger energy density of the mixtures as the fraction of small spheres increases. From the curves in Fig. 5 we extract the value of the strain at the peak, $\gamma_{\text {peak }}$ and the magnitude of the stress overshoot $\sigma_{\text {peak }} / \sigma_{\text {steady }}-1$ to quantify the stress overshoot as a function of $x_{\mathrm{s}}$ and $\mathrm{Pe}_{\dot{\gamma}}$. For one-component hard-sphere glasses $\left(x_{\mathrm{s}}=0\right.$ and 1$)$ this stress response, in particular the stress overshoot, has previously been observed and studied as a function of $\mathrm{Pe}_{\dot{\gamma}}{ }^{\mathbf{9 1 3 - 1 5 , 4 8}}$ It has been associated with the maximal cage distortion before the cage breaks. ${ }^{9,13}$ During cage distortion stress is stored, and is only released when the deformation of the cage is partially relaxed by out-of-cage motion, resulting in the overshoot. Moreover, the overshoot is linked to superdiffusive particle motion observed in experiments and simulations, and predicted by mode coupling theory. ${ }^{13-15}$

The strain at the overshoot, $\gamma_{\text {peak }}$, is associated with the yield strain. It exhibits a dependence on composition $x_{\mathrm{s}}$, which is comparable for all $\mathrm{Pe}_{\dot{\gamma}}$ (Fig. 6a). The yield strain $\gamma_{\text {peak }}$ initially decreases until it reaches a minimum at $x_{\mathrm{s}}=0.3$ and then increases again. This $x_{\mathrm{s}}$ dependence reflects the $x_{\mathrm{s}}$ dependence of the number-averaged long-time structural relaxation time $\left\langle\tau^{\text {long }}\right\rangle$ (Fig. 3), which is associated with the distance to the glass transition. This suggests that the yield strain is larger for systems 


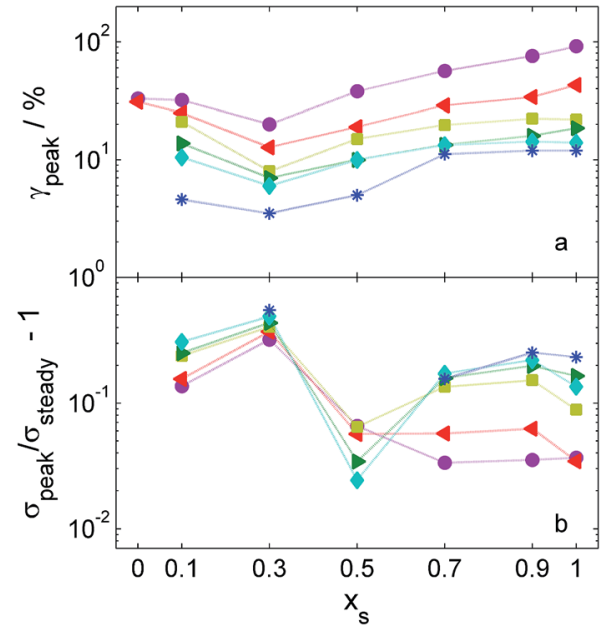

Fig. 6 (a) Strain at the stress overshoot, $\gamma_{\text {peak, }}$ which can be taken as the yield strain, and (b) magnitude of the stress overshoot, $\sigma_{\text {peak }} / \sigma_{\text {steady }}$ -1 , as a function of composition $x_{\mathrm{s}}$ for Péclet numbers $\mathrm{Pe}_{\dot{\gamma}}=0.03(*)$, $0.24(\$), 0.64(\downarrow), 1.20(\square), 2.40(\varangle)$ and $4.70(\bullet)$.

which are deeper in the glass state. It might also be related to variations in the localisation length of the caging species.

In samples for which a broad range of $\mathrm{Pe}_{\dot{\gamma}}$ values is explored, namely $x_{\mathrm{s}}=0.5$ and 0.7 , two regimes in the $\mathrm{Pe}_{\dot{\gamma}}$ dependence of the yield strain $\gamma_{\text {peak }}$ are observed (Fig. 7a). The yield strain $\gamma_{\text {peak }}$ remains approximately constant at $\gamma_{\text {peak }} \approx 10 \%$ for $\mathrm{Pe}_{\dot{\gamma}} \lesssim 1$, in agreement with MCT predictions for one-component glasses, ${ }^{48}$ but increases for larger $\mathrm{Pe}_{\dot{\gamma}}$, similar to experimental results on one-component colloidal glasses of hard-sphere like particles. ${ }^{9,15}$ This behaviour becomes clearer by rescaling the yield strain $\gamma_{\text {peak }}$ with a scaling factor $Z^{\prime}\left(x_{\mathrm{s}}\right)$ (Fig. 7 , inset), which is the average of the $\gamma_{\text {peak }}$ values obtained for the different Pe values at a given composition $x_{\mathrm{s}}$ (Fig. 6a). As expected, the scaling factor $Z^{\prime}\left(x_{\mathrm{s}}\right)$ (Fig. 8) follows the $x_{\mathrm{s}}$ dependence of $\gamma_{\text {peak }}$ and hence also $\left\langle\tau^{\text {long }}\right\rangle$, similar to the data in Fig. 6 a.

The behaviour in the two regimes can be understood by considering the relevant time scales; the characteristic time scale of shear, $\tau_{\text {shear }}=1 / \dot{\gamma}$, and the inherent time scale of the sample, namely the number-averaged short-time Brownian time $\left\langle\tau^{\text {short }}\right\rangle$ (defined in Section 3.2). If $\left.\tau_{\text {shear }}\right\rangle\left\langle\tau^{\text {short }}\right\rangle$, i.e. $\mathrm{Pe}_{\dot{\gamma}}<1$, the shear-induced deformation is slow compared to the Brownian dynamics. Therefore structural rearrangements and yielding can occur once the shear-induced cage deformation is sufficiently large to facilitate escape through Brownian motion. This cage deformation is expected to be similar to the size of the cage in a glass or dense fluid (Fig. 2, inset), consistent with the observed $\gamma_{\text {peak }} \approx 10 \%$. At larger shear rates $\dot{\gamma}$, when $\tau_{\text {shear }} \lesssim$ $\left\langle\tau^{\text {short }}\right\rangle$ or equivalently $\mathrm{Pe}_{\dot{\gamma}} \gtrsim 1$, the probability of cage escape due to Brownian motion decreases. With increasing $\mathrm{Pe}_{\dot{\gamma}}$, the particle displacements are increasingly dominated by the affine motion imposed by shear while the contribution by (random) Brownian motion decreases and thus particle collisions become less probable. Therefore, before yielding occurs the cage is deformed more, i.e. $\gamma_{\text {peak }}$ increases. The rescaled yield strain $\gamma_{\text {peak }} / Z^{\prime}$ is found to increase linearly with $\mathrm{Pe}_{\dot{\gamma}}$ for $\mathrm{Pe}_{\dot{\gamma}} \gtrsim 1$ (Fig. 7a, inset). Thus $\gamma_{\text {peak }}=\dot{\gamma} t_{\text {peak }}=0.1 \mathrm{Pe}_{\dot{\gamma}}=0.1 \dot{\gamma}\left\langle\tau^{\text {short }}\right\rangle$ and
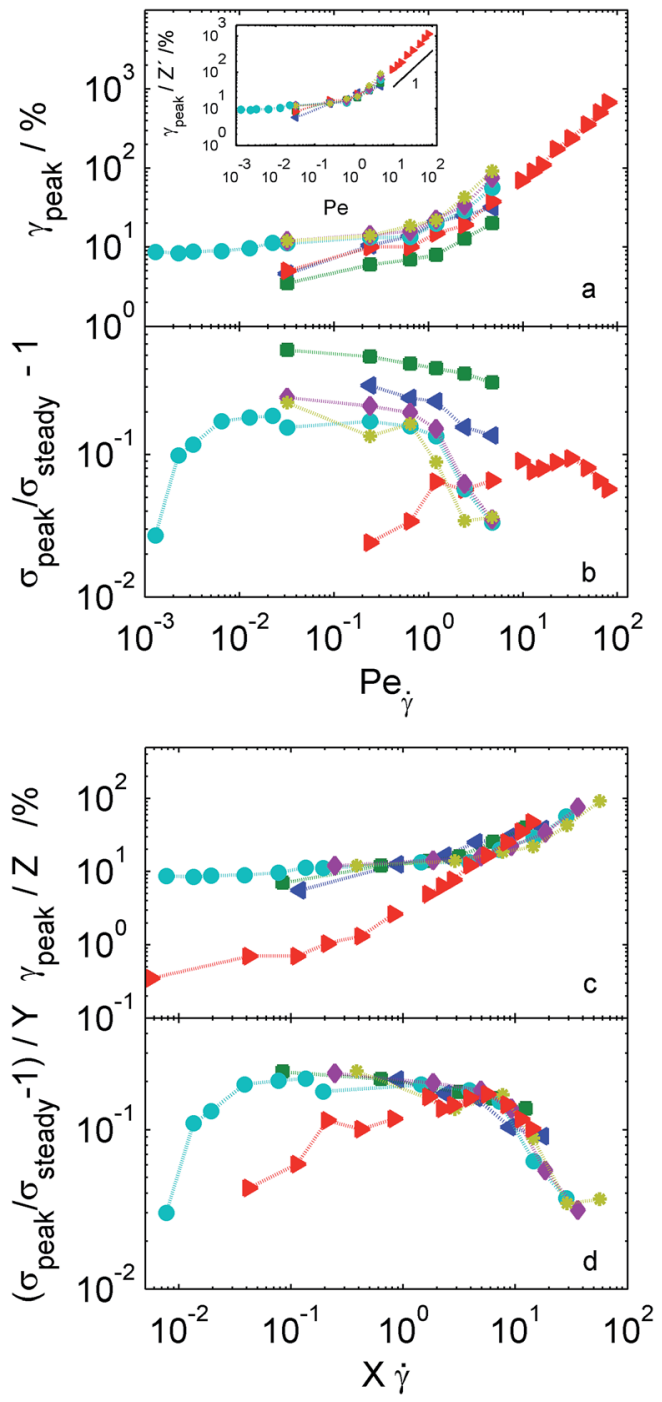

Fig. 7 (a) Strain at the stress overshoot, $\gamma_{\text {peak }}$, and (b) magnitude of the stress overshoot, $\sigma_{\text {peak }} / \sigma_{\text {steady }}-1$, as a function of Péclet number $\mathrm{Pe}_{\dot{\gamma}}$ and $(c)$ rescaled yield strain, $\gamma_{\text {peak }} / Z\left(x_{\mathrm{s}}\right)$, and (d) rescaled magnitude of the stress overshoot, $\left(\sigma_{\text {peak }} / \sigma_{\text {steady }}-1\right) / Y\left(x_{s}\right)$, as a function of rescaled shear rate, $X\left(x_{\mathrm{s}}\right) \dot{\gamma}$, for compositions $x_{\mathrm{s}}=0.1(\Delta), 0.3(\square), 0.5(\downarrow), 0.7(\bullet)$, $0.9(\diamond), 1.0(*)$. The data in (c) and (d) are the same as in (a) and (b), respectively. The inset to (a) shows the same data as in the main plot, but superimposed along the ordinate using the scaling factor $Z^{\prime}\left(x_{s}\right)$. The line indicates a slope of 1 . (See text for details on the rescaling.)

hence $t_{\text {peak }}=0.1\left\langle\tau^{\text {short }}\right\rangle$. Therefore, independent of $\dot{\gamma}$ or, equivalently, $\mathrm{Pe}_{\dot{\gamma}}$, yielding occurs after the same time, about $0.1\left\langle\tau^{\text {short }}\right\rangle$. This suggests that for yielding to occur, at least a shear-induced (affine) displacement of about $10 \%$ and a minimum Brownian (random) displacement are required. The minimum mean squared displacement $\delta y_{\text {peak }}{ }^{2}=2 D^{\text {shear }} t_{\text {peak }}=$ $2 D^{\text {shear }} 0.1\left\langle\tau^{\text {short }}\right\rangle \leqq 0.2\left\langle R^{2}\right\rangle$, where the last relation provides an upper boundary since the diffusion coefficient under shear, $D^{\text {shear }}$ (Section 3.5), is smaller than the one in the quiescent state, which is implicitly contained in $\left\langle\tau^{\text {short }}\right\rangle$. The minimum displacement hence is about the size of the cage. A more quantitative comparison needs to consider the anisotropic structure of the sheared cages. ${ }^{9,13}$ 


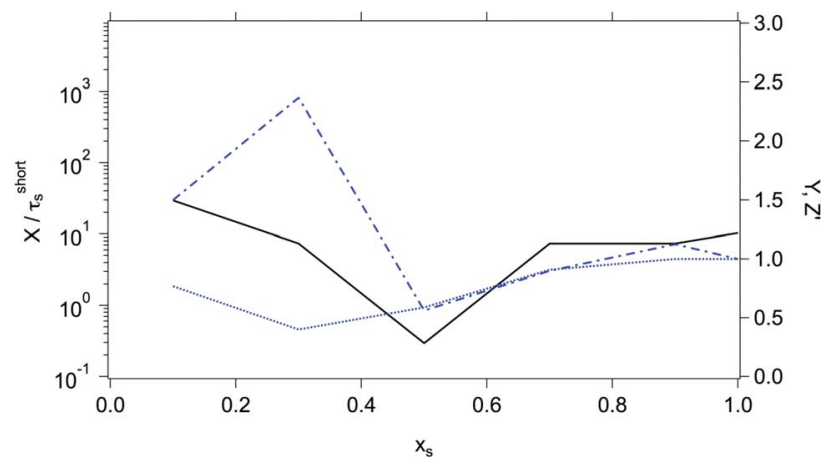

Fig. 8 Composition dependence of the scaling factors of the shear rate, $X$ (solid line), of the strain at the stress overshoot, $Y$ (dasheddotted line), and of the magnitude of the stress overshoot, $Z^{\prime}$ (dotted line). The scaling factor $X$ represents a characteristic time and is normalized by the short-time Brownian time of the small spheres $\tau_{\mathrm{s}}^{\text {short }}$. (For details on the scaling factors see text.)

Two regimes are also observed for the shear rate dependence of the magnitude of the stress overshoot, quantified by $\sigma_{\text {peak }} /$ $\sigma_{\text {steady }}-1$, for $x_{\mathrm{s}}=0.5$ and 0.7 (Fig. 7b). At small $\mathrm{Pe}_{\dot{\gamma}}$, the magnitude of the stress overshoot increases with increasing $\mathrm{Pe}_{\dot{\gamma}}$, as already observed in experiments on thermosensitive pNIPAM particles and as predicted by MCT for one-component systems. ${ }^{48}$ It then reaches a maximum and decreases for large $\mathrm{Pe}_{\dot{\gamma}}$, similar to one-component glasses of hard-sphere like PMMA particles.,99 The transition between the two regimes occurs at transitional Péclet numbers which depend on $x_{\mathrm{s}}$, in contrast to the dependence of $\gamma_{\text {peak }}$ on Pe. In particular, the $\sigma_{\text {peak }} / \sigma_{\text {steady }}-1$ dependence for $x_{\mathrm{s}}=0.5$ (Fig. $7 \mathrm{~b}, \triangleright$ ) is shifted to considerably larger values of $\mathrm{Pe}_{\dot{\gamma}}$ compared to dependencies observed for other $x_{\mathrm{s}}$. That the transitional Péclet number depends on $x_{\mathrm{s}}$ implies that the time at which the transition occurs does not scale with the composition-averaged short-time Brownian time $\left\langle\tau^{\text {short }}\right\rangle$, which determines $\mathrm{Pe}_{\dot{\gamma}}$.

To determine the appropriate characteristic time of the transition in $\sigma_{\text {peak }} / \sigma_{\text {steady }}-1$ as a function of $x_{\mathrm{s}}$, the data in Fig. $7 \mathrm{~b}$ are rescaled as $\left(\sigma_{\text {peak }} / \sigma_{\text {steady }}-1\right) / Y\left(x_{\mathrm{s}}\right)$ versus $X\left(x_{\mathrm{s}}\right) \dot{\gamma}$, where the scaling factors $X\left(x_{\mathrm{s}}\right)$ and $Y\left(x_{\mathrm{s}}\right)$ are chosen such that the resulting curves superimpose (Fig. 7d), that is the curves are shifted horizontally such that the transition occurs at $X\left(x_{\mathrm{s}}\right) \dot{\gamma}=1$ and vertically that the curves overlap. The scaling factor $X\left(x_{\mathrm{s}}\right)$ hence represents the characteristic time of the transition between the increasing and the decreasing branches of $\sigma_{\text {peak }} /$ $\sigma_{\text {steady }}-1$ for the different $x_{\mathrm{s}}$. It exhibits a pronounced minimum at $x_{\mathrm{s}}=0.5$ (Fig. 8 , solid line). The $x_{\mathrm{s}}$ dependence is thus qualitatively different from the monotonously decreasing $\left\langle\tau^{\text {short }}\right\rangle$. However, the dependence appears similar to the one of the dominant structural relaxation time in the quiescent state, $\tilde{\tau}^{\text {long }}$ (Fig. 3, solid line), which is the relaxation time of the relevant caging species, i.e. the large particles for $x_{\mathrm{s}} \leq 0.3$ and the small particles for $x_{\mathrm{s}}>0.3$.

Therefore, the transition between the two regimes depends on the balance between $\tau_{\text {shear }}$ and the dominant structural relaxation time $\bar{\tau}^{\text {long }}$. This indicates that the processes relevant for stress transmission involve particle movements on length scales of out-of-cage diffusion. This is consistent with the fact that in one-component systems the overshoot has been associated with the yielding of the cage.,9,13 The out-of-cage movements are longer than those required for cage deformation, which determine $\gamma_{\text {peak }}$, and hence the timescale of out-of-cage diffusion is not relevant for the transition between the two regimes of the Péclet number dependence of $\gamma_{\text {peak }}$. This is supported by the poor overlap of the $\gamma_{\text {peak }}$ curves if scaled by the same $X\left(x_{\mathrm{s}}\right)$ used for scaling the stresses (Fig. 7c). The overlap is not significantly improved by also scaling $\gamma_{\text {peak }}$ by $Z\left(x_{\mathrm{s}}\right)$ such that all curves superimpose in the ordinate and on the right branch of the curve with $x_{\mathrm{s}}=1.0$ in the abscissa (Fig. 7c).

The value of $Y\left(x_{\mathrm{s}}\right)$ (Fig. 8) corresponds to the average value of $\sigma_{\text {peak }} / \sigma_{\text {steady }}-1$ for a given $x_{\mathrm{s}}$. The magnitude of the overshoot, $\sigma_{\text {peak }} / \sigma_{\text {steady }}-1$ (Fig. 6b) increases from $x_{\mathrm{s}}=0.1$, attains a maximum at $x_{\mathrm{s}}=0.3$ and reaches a minimum at $x_{\mathrm{s}}=0.5$. Subsequently it stays about constant for large $\mathrm{Pe}_{\dot{\gamma}}$ (2.40 to 4.70) or increases to an also approximately constant value for small $\mathrm{Pe}_{\dot{\gamma}}(0.03$ to 1.20$)$. The difference between small and large $\mathrm{Pe}_{\dot{\gamma}}$ is related to the two regimes of the stress response discussed above (Fig. 7a and b).

\subsection{Dynamics under shear}

We aim to link the effects observed in the rheological measurements to the individual-particle dynamics under shear determined by confocal microscopy. Confocal microscopy allows us to image colloids during the step rate experiments and hence to follow shear-induced changes in the dynamics of the large particles, which are fluorescently labelled. Based on the particle trajectories in the velocity-vorticity plane, $\left(x_{i}(t), y_{i}(t)\right)$, transient mean squared displacements in the vorticity direction, $\delta y^{2}$, are calculated for different waiting times $t_{\mathrm{w}}$ after application of shear:

$$
\delta y^{2}\left(t, t_{\mathrm{w}}\right)=\left\langle\left(y_{i}\left(t+t_{\mathrm{w}}\right)-y_{i}\left(t_{\mathrm{w}}\right)\right)^{2}\right\rangle_{i}
$$

where the average runs over all large particles $i$ in the field of view, but not the waiting time $t_{\mathrm{w}}$ (eqn (1)). In the vorticity (neutral) direction contributions of affine particle motions are absent, and thus do not affect an investigation of the effects of shear on the Brownian motion of the particles. The particle dynamics can only reliably be determined using particle tracking if the particles move less than about a tenth of their radius between two successive frames. This limits the shear rates $\dot{\gamma}$ or Péclet numbers $\mathrm{Pe}_{\dot{\gamma}}$ to $10^{-2}<\mathrm{Pe}_{\dot{\gamma}}<1$, which corresponds to the regime where Brownian motion significantly contributes to yielding and stress relaxation (Fig. 7a and b).

After shear is switched on, a steady-state develops. The corresponding MSDs in the steady-state are reported in Fig. 9 (thick color lines), together with the MSDs in the quiescent state (thick black lines). Compared to the quiescent state, the steady-state MSDs exhibit stronger localization at short times, but also faster long-time dynamics, namely a significantly increased long-time diffusion coefficient $D_{\mathrm{L}}^{\text {steady }}$, which increases with increasing $\mathrm{Pe}_{\dot{\gamma}}$ for all compositions $x_{\mathrm{s}}$ (Fig. 10a). The increase in $D_{\mathrm{L}}^{\text {steady }}$ corresponds to shear thinning and is in agreement with previous studies on one-component glasses ${ }^{\mathbf{8 , 9 , 1 4 , 1 5 , 5 0}}$ and measurements 


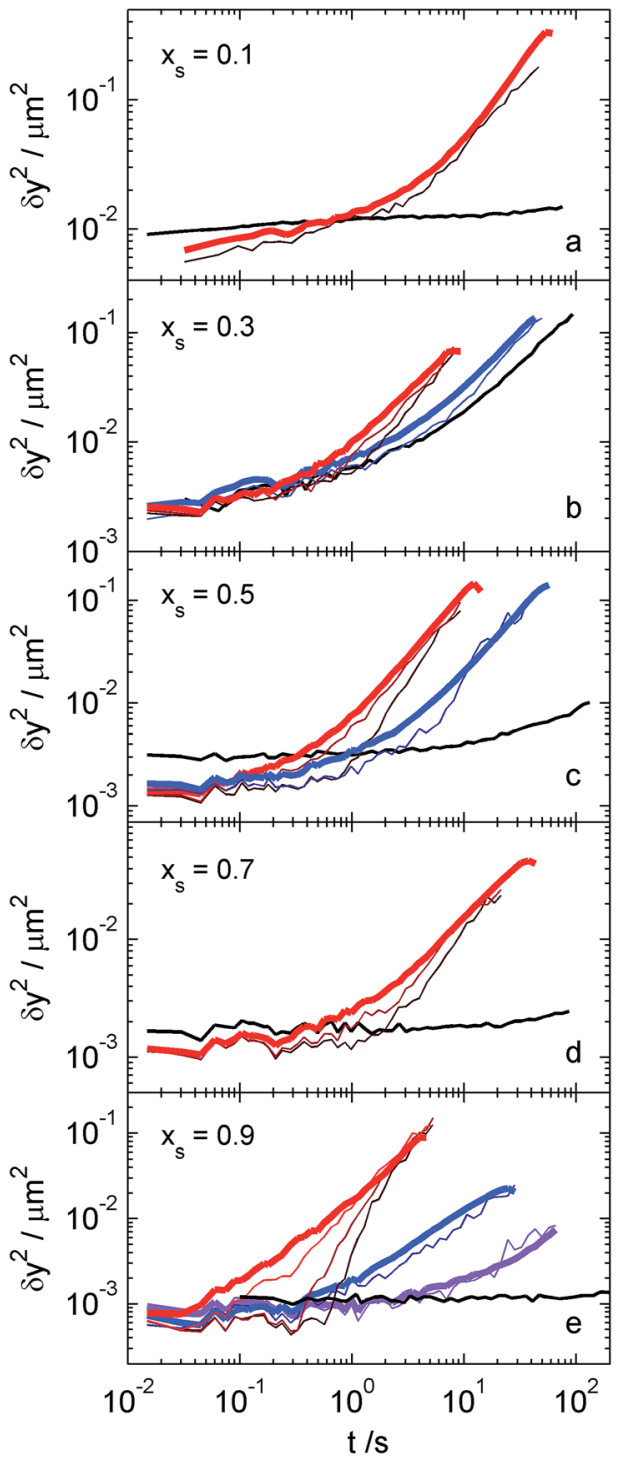

Fig. 9 Mean squared displacement in the vorticity direction $\delta y^{2}$ for different compositions $x_{\mathrm{s}}$ and Péclet numbers. (a) $x_{\mathrm{s}}=0.1, \mathrm{Pe}_{\dot{\gamma}}=0.24$ (red), (b) $x_{\mathrm{s}}=0.3, \mathrm{Pe}_{\dot{\gamma}}=0.24$ (red), 0.08 (blue), (c) $x_{\mathrm{s}}=0.5, \mathrm{Pe}_{\dot{\gamma}}=0.24$ (red), 0.005 (blue), (d) $x_{\mathrm{s}}=0.7, \mathrm{Pe}_{\dot{\gamma}}=0.035$, and (e) $x_{\mathrm{s}}=0.9, P \mathrm{e}_{\dot{\gamma}}=0.28$ (red), 0.028 (blue), 0.003 (violet). The black lines correspond to the MSDs in the quiescent state, thick lines to the MSDs in the steady-state, and thin lines to transient MSDs at waiting time $t_{\mathrm{w}}=0$ and, where present, at longer $t_{\mathrm{w}}$, increasing from bottom to top.

of a two-component glass with $\delta=0.2$ and $x_{\mathrm{s}}=0.9 .{ }^{19}$ For the largest $\mathrm{Pe}_{\dot{\gamma}}$ values, $D_{\mathrm{L}}^{\text {steady }}$ as a function of $x_{\mathrm{s}}$ presents a weak maximum, and hence the fastest shear-induced dynamics, at $x_{\mathrm{s}}=0.3$ (Fig. 10a). The same composition also exhibits the fastest long-time dynamics of the large particles in the quiescent state (Fig. 2 and 3). In addition, this composition shows the smallest $\gamma_{\text {peak }}$ (Fig. 6a), which indicates a link between facilitated yielding, i.e. a smaller yield strain, and fast dynamics in the steady-state, i.e. a larger diffusion coefficient. This is consistent with the observation that yielding requires a minimum mean squared displacement, which is reached earlier for faster dynamics. For the group of data at smaller $\mathrm{Pe}_{\dot{\gamma}}$,

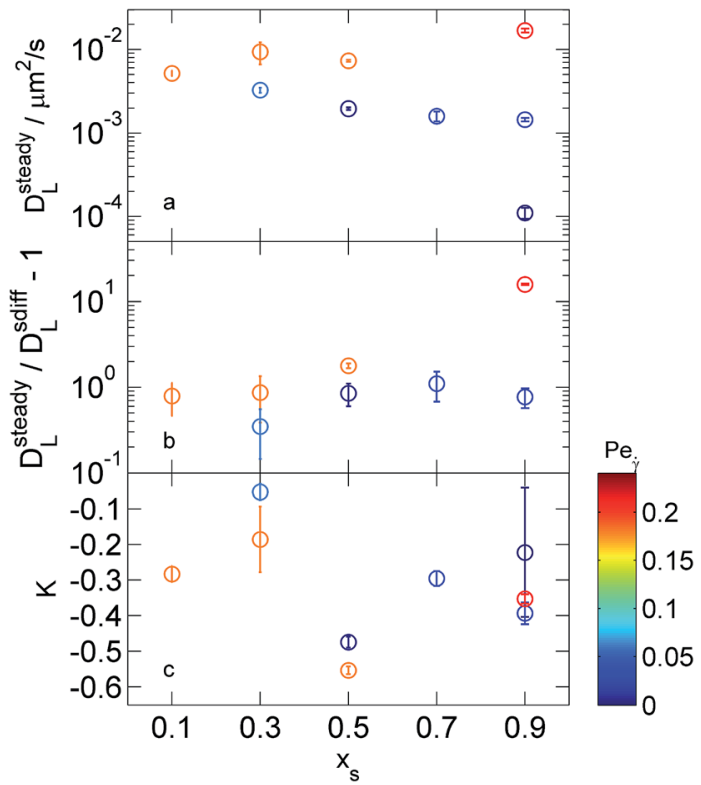

Fig. 10 (a) Steady-state diffusion coefficient $D_{\mathrm{L}}^{\text {steady }}$ of the large spheres, (b) amount of superdiffusion $D_{\mathrm{L}}^{\text {steady }} / D_{\mathrm{L}}^{\text {sdiff }}-1$ of the large spheres at waiting time $t_{\mathrm{w}}=0$, and (c) magnitude of the cage compression $K=\delta y_{\text {shear }}{ }^{2} / \delta y_{\text {rest }}{ }^{2}-1$, as a function of $x_{s}$. Different $\mathrm{Pe}_{\dot{\gamma}}$ values are indicated according to the color scale. The error bars represent variations between repeated measurements with same $x_{\mathrm{s}}$ and $\mathrm{Pe}_{\dot{\gamma}}$.

$D_{\mathrm{L}}^{\text {steady }}$ slightly decreases for $x_{\mathrm{s}} \geq 0.3$, i.e. the steady-state dynamics slows down with increasing $x_{\mathrm{s}}$. This seems to be consistent with the slow-down of the dynamics in the quiescent state and corresponds to the increase of $\gamma_{\text {peak }}$ (Fig. 6a), in agreement with the proposed link between yielding and dynamics in the steady-state.

In addition to the steady-state, the transient state following switch-on of shear is investigated (Fig. 9, thin color lines). At short delay times the transient MSDs moderately increase, associated with a slight expansion of the cage, but they remain below the quiescent MSD indicating tighter localization. At long delay times, and for all waiting times, we observe relatively fast diffusion, already with the steady-state diffusion coefficient $D_{\mathrm{L}}^{\text {steady }}$. While $D_{\mathrm{L}}^{\text {steady }}$ is reached already at the shortest waiting time $t_{\mathrm{w}}$, it is reached at a relatively late delay time $t$, which becomes increasingly shorter as $t_{\mathrm{w}}$ increases. The steady-state MSDs are recovered after a waiting time $t_{\mathrm{w}}^{*}$ which depends on the mixing ratio $x_{\mathrm{s}}$, and has apparently no relation with $\tau_{\text {shear }}$, different from one-component systems. ${ }^{13-15}$

At intermediate delay times a super-linear increase of the MSDs is observed which indicates superdiffusion. The time range with superdiffusion progressively disappears as $t_{\mathrm{w}}$ increases, but also depends on $\mathrm{Pe}_{\dot{\gamma}}$ and $x_{\mathrm{s}}$. The amount of superdiffusion is quantified by $D_{\mathrm{L}}^{\text {steady }} / D_{\mathrm{L}}^{\text {sdiff }}-1$ with $D_{\mathrm{L}}^{\text {sdiff }}$ the apparent diffusion coefficient at maximum superdiffusion, estimated from the minimum of $\delta y^{2} / t v s$. $t$ (not shown). With increasing $x_{\mathrm{s}}$, the amount of superdiffusion, $D_{\mathrm{L}}^{\text {steady }} / D_{\mathrm{L}}^{\text {sdiff }}-1$ increases for (almost) constant, large $\mathrm{Pe}_{\dot{\gamma}}\left(\mathrm{Pe}_{\dot{\gamma}}=0.24\right.$ for $x_{\mathrm{s}}=$ $0.1,0.3,0.5$ and $\mathrm{Pe}_{\dot{\gamma}}=0.28$ for $x_{\mathrm{s}}=0.9$, Fig. 10b orange/red 
color). As expected, this does not reflect the dependence of the stress overshoot, $\sigma_{\text {peak }} / \sigma_{\text {steady }}-1$ (Fig. 6b), since the large particles, whose dynamics is studied here, dominate the rheological response only for $x_{\mathrm{s}} \lesssim 0.5$ (Section 3.4). However, the increase in $D_{\mathrm{L}}^{\text {steady }} / D_{\mathrm{L}}^{\text {sdiff }}-1$ with $x_{\mathrm{s}}$ might reflect the decrease of the localisation length at rest (Fig. 2, inset). This suggests that a tighter localisation at rest leads to a more abrupt and pronounced transition to flow once shear sufficiently deforms the cage to allow particles to escape. The increase of the degree of super-diffusion with increasing $x_{\mathrm{s}}$ seems to become more pronounced with increasing $\mathrm{Pe}_{\dot{\gamma}}$ (Fig. 10b). With increasing $\mathrm{Pe}_{\dot{\gamma}}$, $D_{\mathrm{L}}^{\text {steady }} / D_{\mathrm{L}}^{\text {sdiff }}-1$ increases for all $x_{\mathrm{s}}$ and $t_{\mathrm{w}}=0 \mathrm{~s}$ (Fig. $10 \mathrm{~b}$, different colors). The Pe dependence is similar to the one of $D_{\mathrm{L}}^{\text {steady }}$ and the magnitude of the stress overshoot, $\sigma_{\text {peak }} / \sigma_{\text {steady }}-$ 1 (Fig. 7b). This is consistent with the idea that $\sigma_{\text {peak }} / \sigma_{\text {steady }}-1$ is related to the probability of particle collisions, which occur more frequent as the dynamics becomes faster. Furthermore, it suggests that a larger stored stress results in a more pronounced super-diffusive response, in agreement with similar findings for one-component systems. ${ }^{15}$

At short delay times ( $t \lesssim 1 \mathrm{~s}$, range decreasing with increasing $t_{\mathrm{w}}$ ), the MSDs are dominated by caging (Fig. 9). At these times, the transient MSDs under shear remain below the quiescent state, although they slightly increase with waiting time $t_{\mathrm{w}}$ toward the steady-state. Thus, shear results in a stronger localisation of the large particles in the vorticity direction. The magnitude of cage compression in the vorticity direction is quantified by $K=\delta y_{\text {shear }}^{2} / \delta y_{\text {rest }}^{2}-1$, where $\delta y_{\text {shear }}^{2}$ and $\delta y_{\text {rest }}^{2}$ are the value of the MSD under shear and at rest, respectively, at the same time $0.015 \mathrm{~s} \leq t \leq 0.030 \mathrm{~s}$ (Fig. 10c). The magnitude of the cage compression, $|K|$ decreases from $x_{\mathrm{s}}=0.1$ to 0.3 and 0.5 to 0.9 . Increasing $x_{\mathrm{s}}$ from 0.1 to 0.3 , and from 0.5 to 0.9 , the localization length of the large spheres at rest decreases (Fig. 2a, inset). This implies that the cage is tighter and a smaller free volume is available for compressing the cage, accordingly $|K|$ decreases. However, at $x_{\mathrm{s}}=0.5$, the cage is strongly compressed although the localisation length at $x_{\mathrm{s}}=0.5$ is comparable to that at $x_{\mathrm{s}}=0.3$ in the quiescent state (Fig. 2, inset). Nevertheless, for $x_{\mathrm{s}}=0.5$ the cage is composed of small spheres which might easier rearrange under shear and closely pack around the large spheres than large spheres can. This supports the suggestion that a qualitative change in caging occurs at $x_{\mathrm{s}} \approx 0.5$.

Moreover, $K$ closely resembles the stress overshoot, $\sigma_{\text {peak }} /$ $\sigma_{\text {steady }}-1$ (Fig. 6b), with both exhibiting only a limited dependence on $\mathrm{Pe}_{\dot{\gamma}}$ (within the limited range of $\mathrm{Pe}_{\dot{\gamma}}$ investigated by confocal microscopy). In particular, a large $|K|$ corresponds to a small $\sigma_{\text {peak }} / \sigma_{\text {steady }}-1$ and vice versa. This suggests that stress is partially released through irreversible cage compression, resulting in a smaller stress overshoot. In contrast, if stress can not sufficiently be released through cage compression, it is stored in the system. This storage of stress requires particle movements beyond the cage size and involves several particles. These large movements are related to the long-time diffusion of the cage particles. Hence the relevant timescale is the dominant long-time structural relaxation time $\tilde{\tau}^{\text {long }}$, consistent with the conclusions based on the $x_{\mathrm{s}}$ dependence of $\sigma_{\text {peak }} / \sigma_{\text {steady }}-1$ (Section 3.4). This illustrates the importance of caging and the transition in caging. In contrast, yielding requires many particles to move, although each particle might only move on the length scale of the cage. Moreover, the yield strain $\gamma_{\text {peak }}$ is a relative, dimensionless quantity and hence insensitive to whether the cage is formed by large or small spheres.

\section{Conclusions}

The addition of a second species to a one-component glass results in the loosening of the cage. The transition between caging by small and large particles, respectively, occurs at $x_{\mathrm{s}} \approx$ $0.5 .^{19,20}$ The degree of arrest is reflected in the dynamics at rest, ${ }^{19,20}$ and, as shown here, also under shear. We have shown that under both conditions, at rest and under shear, the mobility is maximum at $x_{\mathrm{s}} \approx 0.3$ (Fig. 2 and 10a).

The change in caging also affects the shear-induced cage compression in vorticity direction, with the strongest compression at $x_{\mathrm{s}} \approx 0.5$ (Fig. 10c). This is attributed to the high mobility of the small particles at $x_{\mathrm{s}} \approx 0.5$ allowing them to realize their higher packing ability in the mixtures. In addition to this particular behaviour, in general the cage compression decreases upon addition of small spheres, which is attributed to an increasingly tighter cage at rest that leaves space for small cage compressions only (Fig. 2, inset). A tight localisation at rest results in an abrupt and pronounced transition to flow once shear-induced cage deformations allow particles to escape. This transition is characterised by transient superdiffusion (Fig. 9 and 10b).

Yielding appears to require Brownian motion beyond a minimum excursion. When this excursion is reached depends on the composition-averaged dynamics of the samples and the shear rate. Slow glassy dynamics thus results in larger yield strains $\gamma_{\text {peak }}$, which is found to increase linearly with the shear rate as long as $\dot{\gamma}\left\langle\tau^{\text {short }}\right\rangle \gtrsim 1$ (Fig. 7a, inset). For the Brownian motion to be effective, an affine shear deformation with $\gamma_{\text {peak }} \gtrsim$ $10 \%$ seems necessary, which limits yielding at small shear rates. We therefore suggest that different processes set a lower limit to the yield strain $\gamma_{\text {peak }}$ at small and large shear rates, respectively.

Since stress is released during cage compression, the magnitude of the stress overshoot is inversely related to the degree of compression and the overshoot linked to superdiffusion. Storage of stress requires rearrangements and particle movements which, in contrast to the processes during yielding, extend significantly beyond the cage and thus occur on the structural relaxation time $\tilde{\tau}^{\text {long }}$ of the caging species, that is the large spheres for $x_{\mathrm{s}} \lesssim 0.5$ and the small spheres for $x_{\mathrm{s}} \gtrsim 0.5$.

In future work, the macroscopic rheological behaviour and the microscopic single-particle dynamics need to be related to the evolution of the microscopic structure during the application of shear, similar to the link established in one-component glasses. ${ }^{9}$

\section{Acknowledgements}

We thank A. B. Schofield for the colloidal particles, J. Horbach and P. Chaudhuri for stimulating discussions, and K. J. Mutch for help with the analysis of the experimental data. We acknowledge funding by the Deutsche Forschungsgemeinschaft 
through the Research unit FOR1394 (Project P2), which also supported the visit of R.C.-P. to Düsseldorf, and EU funding through the FP7-Infrastructures ESMI (CP\&CSA-2010-262348). The Edinburgh work was supported by EPSRC grant EP/ J007404/1.

\section{References}

1 E.-J. Donth, The Glass Transition: Relaxation Dynamics in Liquids and Disordered Materials, Springer-Verlag, Berlin Heidelberg, 2001.

2 C. Suryanarayana and A. Inoue, Bulk Metallic Glasses, CRC Press, Taylor and Francis Group, 2011.

3 P. N. Pusey and W. van Megen, Nature, 1986, 320, 340-342.

4 G. Brambilla, D. El Masri, M. Pierno, L. Berthier, L. Cipelletti, G. Petekidis and A. B. Schofield, Phys. Rev. Lett., 2009, 102, 085703.

5 G. Petekidis, A. Moussaid and P. N. Pusey, Phys. Rev. E: Stat., Nonlinear, Soft Matter Phys., 2002, 66, 051402.

6 K. N. Pham, G. Petekidis, D. Vlassopoulos, S. U. Egelhaaf, P. N. Pusey and W. C. K. Poon, Europhys. Lett., 2006, 75, 624-630.

7 K. N. Pham, G. Petekidis, D. Vlassopoulos, S. U. Egelhaaf, W. C. K. Poon and P. N. Pusey, J. Rheol., 2008, 52, 649.

8 R. Besseling, E. R. Weeks, A. B. Schofield and W. C. K. Poon, Phys. Rev. Lett., 2007, 99, 028301.

9 N. Koumakis, M. Laurati, S. U. Egelhaaf, J. F. Brady and G. Petekidis, Phys. Rev. Lett., 2012, 108, 098303.

10 C. Eisenmann, C. Kim, J. Mattsson and D. A. Weitz, Phys. Rev. Lett., 2010, 104, 035502.

11 M. Siebenbürger, M. Fuchs and M. Ballauff, Soft Matter, 2012, 8, 4014-4024.

12 C. Christopoulou, G. Petekidis, B. Erwin, M. Cloitre and D. Vlassopoulos, Philos. Trans. R. Soc., A, 2009, 367, 50515071.

13 K. J. Mutch, M. Laurati, C. P. Amann, M. Fuchs and S. U. Egelhaaf, Eur. Phys. J.: Spec. Top., 2013, 222, 2803.

14 J. Zausch, J. Horbach, M. Laurati, S. U. Egelhaaf, J. M. Brader, T. Voigtmann and M. Fuchs, J. Phys.: Condens. Matter, 2008, 20, 404210.

15 M. Laurati, K. J. Mutch, N. Koumakis, J. Zausch, C. P. Amann, A. B. Schofield, G. Petekidis, J. F. Brady, J. Horbach, M. Fuchs and S. U. Egelhaaf, J. Phys.: Condens. Matter, 2012, 24, 431207.

16 S. Williams and W. van Megen, Phys. Rev. E: Stat., Nonlinear, Soft Matter Phys., 2001, 64, 041502.

17 A. Imhof and J. K. G. Dhont, Phys. Rev. E: Stat. Phys., Plasmas, Fluids, Relat. Interdiscip. Top., 1995, 52, 6344-6357.

18 A. Imhof and J. K. G. Dhont, Phys. Rev. Lett., 1995, 75, 16621665.

19 T. Sentjabrskaja, D. Guu, M. P. Lettinga, S. U. Egelhaaf and M. Laurati, AIP Conf. Proc., 2013, 1518, 206.

20 T. Sentjabrskaja, R. Babaliari, J. Hendricks, M. Laurati, G. Petekidis and S. U. Egelhaaf, Soft Matter, 2013, 9, 45244533.

21 A. Moreno and J. Colmenero, J. Chem. Phys., 2006, 125, 164507.
22 T. Voigtmann and J. Horbach, Phys. Rev. Lett., 2009, 103, 205901.

23 E. López-Sánchez, C. D. Estrada-Álvarez, G. Pérez-Ángel, J. M. Méndez-Alcaraz, P. González-Mozuelos and R. Castañeda-Priego, J. Chem. Phys., 2013, 139, 104908.

24 W. Götze and T. Voigtmann, Phys. Rev. E: Stat., Nonlinear, Soft Matter Phys., 2003, 67, 021502.

25 T. Voigtmann, Europhys. Lett., 2011, 96, 36006.

26 J. Bosse and J. S. Thakur, Phys. Rev. Lett., 1987, 59, 998-1001. 27 J. Bosse and Y. Kaneko, Phys. Rev. Lett., 1995, 74, 4023-4026.

28 L. Sjögren, Phys. Rev. A, 1986, 33, 1254-1260.

29 R. Seyboldt, D. Hajnal, F. Weysser and M. Fuchs, Soft Matter, 2012, 8, 4132-4140.

30 R. Juárez-Maldonado and M. Medina-Noyola, Phys. Rev. E: Stat., Nonlinear, Soft Matter Phys., 2008, 77, 051503.

31 S. R. Williams, I. K. Snook and W. van Megen, Phys. Rev. E: Stat., Nonlinear, Soft Matter Phys., 2001, 64, 021506.

32 G. Foffi, W. Götze, F. Sciortino, P. Tartaglia and T. Voigtmann, Phys. Rev. Lett., 2003, 91, 085701.

33 B. E. Rodriguez, E. W. Kaler and M. S. Wolfe, Langmuir, 1992, 8, 2382.

34 R. Besseling, L. Isa, E. R. Weeks and W. C. K. Poon, Adv. Colloid Interface Sci., 2009, 146, 1-17.

35 P. Ballesta, G. Petekidis, L. Isa, W. C. K. Poon and R. Besseling, J. Rheol., 2012, 56, 1005-1037.

36 J. C. Crocker and D. G. Grier, J. Colloid Interface Sci., 1996, 179, 298-310.

37 A. Yethiraj and A. van Blaaderen, Nature, 2003, 421, 513-517. 38 C. P. Royall, W. C. K. Poon and E. R. Weeks, Soft Matter, 2013, 9, 17-27.

39 T. G. Mason and D. A. Weitz, Phys. Rev. Lett., 1995, 75, 27702773.

40 T. Sentjabrskaja, M. Laurati, S. U. Egelhaaf and Th. Voigtmann, in preparation.

41 R. Higler, J. Appoel and J. Sprakel, Soft Matter, 2013, 9, 5372.

42 T. Hamanaka and A. Onuki, Phys. Rev. E: Stat., Nonlinear, Soft Matter Phys., 2007, 75, 041503.

43 P. Yunker, Z. Zhang and A. G. Yodh, Phys. Rev. Lett., 2010, 104, 015701.

44 W. van Megen, T. C. Mortensen, S. R. Williams and J. Müller, Phys. Rev. E: Stat. Phys., Plasmas, Fluids, Relat. Interdiscip. Top., 1998, 58, 6073-6085.

45 L. López-Flores, P. Mendoza-Méndez, L. E. Sánchez-Díaz, L. L. Yeomans-Reyna, A. Vizcarra-Rendón, G. Pérez-Ángel, M. Chávez-Páez and M. Medina-Noyola, Europhys. Lett., 2012, 99, 46001.

46 T. O. E. Skinner, S. K. Schnyder, D. G. A. L. Aarts, J. Horbach and R. P. A. Dullens, Phys. Rev. Lett., 2013, 111, 128301.

47 R. Besseling, L. Isa, P. Ballesta, G. Petekidis, M. E. Cates and W. C. K. Poon, Phys. Rev. Lett., 2010, 105, 268301.

48 C. P. Amann, M. Siebenbürger, M. Krüger, F. Weysser, M. Ballauff and M. Fuchs, J. Rheol., 2013, 57, 149-175.

49 N. Koumakis, M. Laurati, K. J. Mutch, J. F. Brady, S. U. Egelhaaf and G. Petekidis, in preparation.

50 N. Koumakis, J. F. Brady and G. Petekidis, Phys. Rev. Lett., 2013, 110, 178301. 